Revue internationale P.M.E.

Économie et gestion de la petite et moyenne entreprise

\title{
Classement des stratégies de production des PME d'Alicante
}

\section{Enrique Claver Cortés, Juan Llopis Taverner, Francisco José Conca Flor et Hipólito Molina Manchón}

Volume 13, numéro 2, 2000

URI : https://id.erudit.org/iderudit/1008677ar

DOI : https://doi.org/10.7202/1008677ar

Aller au sommaire du numéro

\section{Éditeur(s)}

Presses de l'Université du Québec

ISSN

0776-5436 (imprimé)

1918-9699 (numérique)

Découvrir la revue

Citer cette note

Claver Cortés, E., Llopis Taverner, J., Conca Flor, F. J. \& Molina Manchón, H. (2000). Classement des stratégies de production des PME d'Alicante. Revue internationale P.M.E., 13(2), 97-125. https://doi.org/10.7202/1008677ar

\section{Résumé de l'article}

Cet article est un essai de classement des stratégies de production des principales entreprises manufacturières de la province d'Alicante en Espagne. Les 75 entreprises étudiées ont été divisées en trois groupes. Le premier groupe, appelé fabricants de produits haut de gamme, englobe les entreprises offrant des produits de haute performance à prix élevé destinés à un marché exigeant et soucieux de la qualité. La conception et la technologie constituent des facteurs importants pour la compétitivité de ces entreprises et leur gestion vise la réduction des coûts plus que y accroissement de la flexibilité. Le deuxième groupe, fabricants de produits de masse à court délai, est capable de satisfaire adéquatement les besoins d'un marché exigeant en ce qui a trait aux prix et à la livraison. La gestion de ce type d'entreprise est principalement basée sur ces caractéristiques, et la capacité d'innovation ainsi que les prix des fournisseurs y sont particulièrement valorisés. Enfin, le troisième groupe, composé des fabricants de produits de masse orientés vers la variété, semble cibler un marché de masse très exigeant au niveau du prix avec un produit mature, tout en donnant l'impression de compenser sa faiblesse en matière de prix en offrant une gamme de produits plus étendue, ce qui incite les gestionnaires à mettre l'accent sur la flexibilité plutôt que sur la réduction des coûts.
Ce document est protégé par la loi sur le droit d'auteur. L'utilisation des services d'Érudit (y compris la reproduction) est assujettie à sa politique d'utilisation que vous pouvez consulter en ligne.

https://apropos.erudit.org/fr/usagers/politique-dutilisation/ 


\title{
Classement des stratégies de production des PME d'Alicante
}

\author{
Enrique CLAVER CORTÉS \\ Juan LLOPIS TAVERNER \\ Francisco José CONCA FLOR \\ Hipólito MOLINA MANCHÓN \\ Université d'Alicante
}

\section{MOTS CLÉS}

\section{Classement - Stratégies de production - PME Entreprises manufacturières - Analyse - Regroupement}

\begin{abstract}
LES AUTEURS
EnRIQue Claver Cortés est licencié et docteur en sciences économiques et de l'entreprise de l'Université de Valence. II est professeur de stratégie patronale de la Faculté de sciences économiques et directeur de l'unité de recherche "Organisation de l'entreprise " de l'Université d'Alicante. Professeur de la chaire d'organisation de l'entreprise, son activité de recherche porte, notamment, sur la stratégie des sociétés et la direction de la technologie.

JUAN LLOPIS TAVERNER est licencié et docteur en sciences économiques et de l'entreprise de l'Université d'Alicante. II est maître de conférences en direction de la production et ressources humaines et vice-doyen secrétaire de la Faculté de sciences économiques et de l'entreprise de l'Université d'Alicante. Son activité de recherche est axée sur la direction des ressources humaines et la direction des entreprises.

FrANCISCO JosÉ CONCA FLOR est licencié et docteur en sciences économiques et de l'entreprise de l'Université d'Alicante. Il est maître de conférences en administration de la production et de la logistique de la Faculté de sciences économiques et de l'École polytechnique supérieure de l'Université d'Alicante. Ses intérêts de recherche sont la stratégie des opérations, la stratégie technologique et la direction logistique de l'entreprise. Courriel: <rancisco.conca@ua.es>

HiPólito Molina ManCHón est licencié et docteur en sciences économiques et de l'entreprise de l'Université d'Alicante. II est maître de conférences en gestion de l'innovation et de la technologie et en organisation de l'entreprise de la Faculté de sciences économiques et de l'École polytechnique supérieure de l'Université d'Alicante. Son activité de recherche vise l'étude de la direction stratégique de l'innovation et la technologie.
\end{abstract}

Adresse: Departamento de Organización de Empresas. Universidad de Alicante. Apartado de Correos 99, 03080 Alicante, España. Téléphone et télécopieur: 965903606. 


\section{RÉSUMÉ}

Cet article est un essai de classement des stratégies de production des principales entreprises manufacturières de la province d'Alicante en Espagne. Les 75 entreprises étudiées ont été divisées en trois groupes. Le premier groupe, appelé fabricants de produits haut de gamme, englobe les entreprises offrant des produits de haute performance à prix élevé destinés à un marché exigeant et soucieux de la qualité. La conception et la technologie constituent des facteurs importants pour la compétitivité de ces entreprises et leur gestion vise la réduction des coûts plus que l'accroissement de la flexibilité. Le deuxième groupe, fabricants de produits de masse à court délai, est capable de satisfaire adéquatement les besoins d'un marché exigeant en ce qui a trait aux prix et à la livraison. La gestion de ce type d'entreprise est principalement basée sur ces caractéristiques, et la capacité d'innovation ainsi que les prix des fournisseurs y sont particulièrement valorisés. Enfin, le troisième groupe, composé des fabricants de produits de masse orientés vers la variété, semble cibler un marché de masse très exigeant au niveau du prix avec un produit mature, tout en donnant l'impression de compenser sa faiblesse en matière de prix en offrant une gamme de produits plus étendue, ce qui incite les gestionnaires à mettre l'accent sur la flexibilité plutôt que sur la réduction des coûts.

\section{ABSTRACT}

This paper offers a taxonomy of the manufacturing strategies of the small and medium manufacturing firms in the province of Alicante (Spain). The seventy-five firms studied were divided into three groups. The first one, which we call highperformance products manufacturers, is characterized by providing expensive, high performance products to a market which demands these features; design and technology are very important to reinforce their competitive advantage, and factory management is more oriented towards reducing costs than towards improving flexibility. Time-focused mass manufacturers are capable of adequately catering for the needs of a market demanding in prices and deliveries; factory management actively supports these characteristics, and they specifically value innovativeness and prices in their suppliers. Finally, although variety-reoriented mass manufacturers seem to address themselves to a mass market with a mature product, it also seems that they try to make up for their weakness concerning prices by means of a wider offer in their product mix. This has an influence upon factory management, where flexibility becomes a more important issue than reducing costs.

\section{RESUMEN}

En el presente artículo se realiza una clasificación de las estrategias de producción de las pequeñas y medianas empresas manufactureras de la provincia de Alicante (España). Las 75 empresas estudiadas se clasificaron en tres grupos. El primero, que denominamos fabricantes de productos de elevadas prestaciones, se caracteriza por ofertar productos caros y de elevadas prestaciones a un mercado exigente en estos aspectos; el diseño y la tecnología son muy importantes para fortalecer su ventaja competitiva ; y la gestión de la fábrica está mas orientada hacia la reducción de costes que a la mejora de la flexibilidad. Los fabricantes en masa centrados en el tiempo son capaces de satisfacer adecuadamente las necesidades de un mercado exigente en precios y entregas; la gestión de la fábrica apoya 
activamente estas características; y valoran especialmente la capacidad innovadora y los precios de sus proveedores. Finalmente, los fabricantes en masa reorientados hacia la variedad, si bien parecen dirigirse a un mercado de masas muy exigente en precios y con un producto maduro, da la impresión de que tratan de compensar su debilidad en precios con una mayor oferta en su gama de productos, lo cual repercute en la gestión de la fábrica donde la flexibilidad se convierte en un problema más importante que la reducción de costes.

\section{ZUSAMMENFASSUNG}

In diesen Artikel nehmen wir eine Klassifizierung von Produktionsstrategien in KMU in der Provinz von Alicante (Spanien). Die 75 zu untersuchenden Unternehmen wurden in drei Gruppen eingeteilt. Die erste Gruppe bezeichnen wir als Hersteller von Markenartikeln, welche sich dadurch charakterisieren, dass sie exklusive Produkte und Dienstleistungen auf einem sehr anspruchsvollen Markt anbieten. Die Technologie und das Design sind sehr wichtige Faktoren um der Konkurrenz dieser ersten Gruppe standzuhalten. Auf der anderen Seite konzentriert sich die Unternehmensführung mehrheitlich auf die Kostenreduktion als auf die Verbesserung der Flexibilität.

Die zweite Gruppe bezeichnen wir als Hersteller von Massenartikeln, welche die Bedürfnisse eines Marktes befriedigen, der angemessene Preise und Fristen verlangt. Die Unternehmensführung konzentriert sich auf diese zwei Charakteristiken, da die Innovationskapazität und die Preisgestaltung der Lieferanten speziell evaluiert werden. Schlussendlich orientieren sich die Hersteller von Massenartikeln auf die Vielfalt von Produkten, obwohl sie einem sehr anspruchsvollen Markt betreffend Preis und Produktereife gegenüberstehen. Dabei haben wir den Eindruck, dass diese Hersteller ihre Schwächen betreffend Produktepreise mit einem grösseren Angebot an Produkten kompensieren, was sich auch auf die Unternehmensführung auswirkt, wo die Flexibilität wichtiger wird als die Kostenreduktion.

\section{Introduction}

De nos jours, l'énorme potentiel de la fonction de production comme source d'avantages concurrentiels est un fait bien constaté chez les grandes entreprises industrielles et tout aussi préconisé par les spécialistes de la direction des opérations. C'est à partir de nombreux concepts déjà existants en accord avec cette ligne de pensée qu'est apparue et s'est consolidée dans les années 1980 une théorie sur la stratégie de production en parfaite cohésion, d'une part, avec les concepts de stratégie de production et, d'autre part, avec une série de caractéristiques propres nous permettant de les différencier des précédentes.

Une partie des recherches effectuées sur la stratégie de production s'est principalement centrée sur l'établissement de typologies ou de stratégies génériques de production à partir de l'observation empirique. L'utilité de ce genre de travaux est manifeste, tant au point de vue du progrès de la recherche dans le domaine qui nous occupe, qu'à celui de la direction de production. Ainsi, le classement fournit des 
descriptions utiles pour la discussion, la recherche et la pédagogie, en plus de permettre de dégager des groupes d'entreprises ayant des profils communs pour mettre au jour les structures sous-jacentes de la compétitivité en ce qui concerne la fonction de production (Miller et Roth, 1994).

Il importe de préciser cependant que ces études portent généralement sur des entreprises de grande dimension appartenant à des secteurs industriels clés tels que l'électronique ou l'automobile. C'est ainsi que nous remarquons, dans les différentes recherches, l'absence de travaux taxinomiques sur les petites et moyennes entreprises, en particulier sur celles qui exercent leurs activités dans des secteurs que nous ne pouvons pas qualifier de «prédominants » sur le plan du développement des techniques de gestion.

Le présent article a pour but de combler cette lacune dans le domaine. Pour ce faire, l'ouvrage a été divisé en six parties en incluant la présente introduction ainsi que les conclusions. Dans la deuxième partie, nous effectuons un survol des différents travaux consacrés à la stratégie de production ainsi qu'un examen de la méthodologie et des études taxinomiques, tout en proposant certaines modifications permettant de les appliquer aux PME. Dans la troisième partie, nous exposons la méthodologie du travail empirique. Nous établissons ensuite le classement des entreprises en trois groupes dans la quatrième partie et, dans la cinquième, nous discutons des résultats obtenus. Dans la conclusion, nous revenons sur ces résultats en montrant leurs limites et en relevant des interrogations à explorer dans des recherches ultérieures.

\section{Recension des études antérieures}

L'analyse des différentes études ${ }^{1}$ portant sur la stratégie de production révèle que la majorité des auteurs, qui se sont inspirés des premières recherches de Skinner (1969) et de Wheelwright (1978), s'entendent pour proposer un modèle qui comprendrait, d'un côté, la détermination des priorités compétitives de production - en définissant ce que nous appelons « tâche » de production - et, de l'autre, l'établissement des principales politiques à adopter.

En partant de ce modèle conceptuel, une série de travaux taxinomiques ont été réalisés pour établir une typologie de production, en prenant comme référence la «tâche » de production pour établir le classement et en vérifiant par la suite les

1. Nous avons analysé les travaux de Beckman et al. (1990), Bolwijn et Kumpe (1990), Buffa (1984), De Meyer et Wittenberg-Cox (1994), De Meyer et al. (1989), Fine et Hax (1985), Garvin (1993), Greenhalgh (1990), Haas (1987), Hayes et Wheelwright (1984), Hayes, Weelwright et Clark (1988), Hill (1993), Leong, Snyder et Ward (1990), Miller (1988), Mills, Platts et Gregory (1995), Platts et Gregory $(1990,1992)$, Schroeder et Lahr (1990), Skinner (1985) et Voss $(1989,1992)$. 
politiques de production suivies par chacun des groupes identifiés (De Meyer, 1992; Miller et Roth, 1998, 1994 ; Richardson, Taylor et Gordon, 1985 ; Stobaugh et Telesio, 1983 ; Sweeney et Szwejczewski, 1996; et, en Espagne, Avella, Fernández et Vázquez, 1996).

Cependant, cette perspective pose le problème suivant : lorsque vient le temps de considérer les priorités compétitives afin de déterminer la « tâche de production », nous incluons généralement dans cette catégorie les objectifs de production (le coût, la flexibilité ou le temps de production) ainsi que les attributs du produit qui sont « visibles » pour le client (le prix, la variété de produits ou le délai de livraison; Swink et Hegarty, 1998 ; Corbett et Wassenhove, 1993). Ainsi, il arrive que certains aspects relevant plutôt de la stratégie des affaires, comme l' " avantage compétitif », soient inclus parmi les variables de classement, puisque nous considérons qu'une entreprise offrant des produits dont les attributs sont supérieurs à ceux de la concurrence possède effectivement un avantage compétitif. Néanmoins, cette façon de procéder nous paraît justifiée pour l'établissement d'une taxinomie des stratégies de production, étant donné que nous considérons que les stratégies fonctionnelles et la stratégie des affaires sont intimement liées et que, dans le cas présent, cette dernière vient se fondre au concept de «tâche » de production, dans le but de faciliter la compréhension de la stratégie des affaires par la direction (Schmenner, 1990). C'est pourquoi nous avons inclus dans les variables proposées pour le classement, en plus des objectifs de production, une série d'attributs du produit visant à informer sur la nature des avantages compétitifs acquis par l'entreprise, grâce à l'évaluation par cette dernière de sa force pour chaque attribut face à un concurrent de même taille (tableau 1).

Pour être conséquents avec ce qui vient d'être exposé, nous ne devons pas oublier que l'avantage compétitif apparaît au moment où une entreprise tire parti de ses compétences distinctives pour obtenir une bonne position sur le marché, supérieure à celle de la concurrence. Le développement de ces compétences est généralement assuré par les différentes fonctions administratives de l'entreprise (production, marketing, finances, etc.) et il est possible de les exploiter sur le marché lorsqu'elles peuvent satisfaire la demande, c'est-à-dire lorsqu'elles coïncident avec ce que nous appelons les «facteurs clés de réussite» (Bamberger, 1989). Ces considérations justifient l'inclusion de deux autres groupes de variables parmi celles sélectionnées pour effectuer le classement (tableau 1): 1) l'importance des différentes fonctions d'entreprises visant à accroître leur compétitivité (orientation fonctionnelle) et 2) les attributs du produit, analysés cette fois suivant l'importance que le client leur accorde lorsqu'il effectue ses achats (demandes).

Le dernier groupe de variables que nous proposons pour réaliser le classement correspond aux objectifs qui guident la sélection et l'évaluation des fournisseurs (tableau 1). Étant donné leur grande importance, nous avons tendance à inclure ces objectifs, d'une part, dans les travaux théoriques et, d'autre part, dans la pratique 


\section{TABleau 1}

Variables proposées pour réaliser le classement

\begin{tabular}{|c|c|c|c|c|c|}
\hline VARIABLES & Moyenne & Médiane & Écart type & Min. & Max. \\
\hline \multicolumn{6}{|l|}{ ORIENTATION FONCTIONNELLEa } \\
\hline Achats & 7,49 & 7 & 1,68 & 3 & 10 \\
\hline Production & 8,45 & 9 & 1,43 & 5 & 10 \\
\hline Finances & 7,63 & 8 & 1,93 & 3 & 10 \\
\hline Design & 7,37 & 8 & 2,88 & 0 & 10 \\
\hline Technologie & 7,39 & 8 & 2,17 & 2 & 10 \\
\hline Commerciale & 8,24 & 8 & 1,92 & 0 & 10 \\
\hline Distribution & 7,36 & 8 & 2,26 & 0 & 10 \\
\hline \multicolumn{6}{|l|}{ DEMANDES $^{b}$} \\
\hline Performances & 7,47 & 8 & 2,27 & 0 & 10 \\
\hline Service après-vente & 4,85 & 5 & 3,88 & 0 & 10 \\
\hline Qualité de conformité & 8,61 & 9 & 1,71 & 0 & 10 \\
\hline Gamme & 7,39 & 7 & 1,89 & 0 & 10 \\
\hline Ponctualité des livraisons & 8,77 & 9 & 1,27 & 5 & 10 \\
\hline Rapidité des livraisons & 8,48 & 9 & 1,51 & 3 & 10 \\
\hline Prix & 8,45 & 9 & 1,93 & 0 & 10 \\
\hline \multicolumn{6}{|l|}{ FORCES } \\
\hline Rapidité des livraisons & 6,77 & 7 & 1,88 & 4 & 10 \\
\hline Ponctualité des livraisons & 6,84 & 7 & 1,93 & 2 & 10 \\
\hline Performances & 6,97 & 7 & 1,76 & 4 & 10 \\
\hline Gamme & 6,69 & 7 & 1,69 & 3 & 10 \\
\hline Prix & 5,05 & 5 & 1,94 & 0 & 10 \\
\hline Qualité de conformité & 6,89 & 7 & 1,62 & 4 & 10 \\
\hline Service après-vente & 6,03 & 5 & 1,68 & 3 & 10 \\
\hline \multicolumn{6}{|l|}{ OBJECTIFS DE PRODUCTION ${ }^{d}$} \\
\hline Accomplir les programmes & 8,43 & 9 & 2,06 & 0 & 10 \\
\hline Réduire les défauts & 8,45 & 9 & 1,64 & 1 & 10 \\
\hline Accroître la flexibilité & 7,43 & 8 & 2,35 & 0 & 10 \\
\hline Réduire les stocks & 7,17 & 8 & 2,52 & 0 & 10 \\
\hline Réduire les coûts & 8,39 & 9 & 1,69 & 3 & 10 \\
\hline Réduire le temps de fabrication & 7,11 & 8 & 2,90 & 0 & 10 \\
\hline \multicolumn{6}{|l|}{ SÉLECTION DES FOURNISSEURS } \\
\hline Qualité & 9,21 & 10 & 1,38 & 0 & 10 \\
\hline Prix & 8,47 & 9 & 1,66 & 0 & 10 \\
\hline Ponctualité & 8,65 & 9 & 1,72 & 0 & 10 \\
\hline Distance géographique & 3,79 & 4 & 3,31 & 0 & 10 \\
\hline Capacité innovatrice & 6,15 & 7 & 3,14 & 0 & 10 \\
\hline Temps de livraison & 7,97 & 8 & 1,92 & 0 & 10 \\
\hline
\end{tabular}

a. Variables qui mesurent l'importance de chaque fonction entrepreneuriale pour la compétitivité de l'entreprise, où le « 0 » signifie «très peu important » et le « 10 », «très important ».

b. Variables qui mesurent l'importance de chaque attribut du produit pour le client, sur une échelle de 11 points.

c. Variables qui mesurent la position de l'entreprise par rapport à un concurrent moyen pour chacun des attributs, sur une échelle de 11 points, où le « 0 » signifie «bien pire», le « 5 », «à peu près pareille» et le « $10 »$, «bien meilleure».

d. Variables qui mesurent la priorité de chaque objectif de production dans la gestion de l'usine, sur une échelle de 11 points.

e. Variables qui mesurent l'importance de chacun des critères employés pour sélectionner et évaluer les fournisseurs, sur une échelle de 11 points. 
administrative de ce que nous appelons la «gestion de la chaîne d'approvisionnement », c'est-à-dire l'intégration des processus clés d'affaire, à partir du fournisseur d'origine jusqu'au consommateur final (Lambert, Cooper et Pagh, 1998). Cela suppose que la vision de l'organisation comme une entité autonome est graduellement remplacée par la reconnaissance du fait que les entreprises doivent coopérer pour pouvoir être concurrentielles (Christopher et Ryals, 1999) et que l'avantage ou le désavantage d'une entreprise individuelle s'explique fréquemment par l'avantage ou le désavantage du réseau de relations qui l'entoure (Dyer et Singh, 1998). Dans le cas des petites entreprises, diverses études ont démontré qu'une grande partie de leur succès peut être attribuée à une gestion en réseau très efficace de leurs systèmes de production (Chen, 1999; Wu, 1994) donnant lieu, dans certains cas et sur le plan territorial, au concept de « district industriel » (Marshall, 1969 ; Becattini, 1989).

Dans les PME, et afin de justifier encore une fois les différents groupes de variables proposées pour effectuer le classement, on remarque que la présence d'un processus déterminé de planification (Matthews et Scott, 1995 ; Robinson et Pearce, 1984) ou encore d'une stratégie de production explicitement définie et différenciée de la stratégie générale est quasi inexistante ; c'est pourquoi la stratégie de production fait souvent partie intégrante de la stratégie générale de l'entreprise (Harrison, 1993).

\section{Méthodologie}

Les données utilisées pour réaliser cette étude proviennent d'une enquête effectuée en 1997 à l'aide d'un questionnaire fermé, rempli lors d'une série d'entrevues personnelles avec les responsables de production des plus grandes entreprises manufacturières de la province d'Alicante ${ }^{2}$. Pour sélectionner l'échantillon d'entreprises de cette étude, nous avons fixé un chiffre d'affaires minimum de 900 millions de pesetas dans le but d'éliminer toutes les entreprises trop petites pour employer une personne possédant un minimum de formation et de spécialisation dans le secteur fonctionnel de la production. Notre échantillon initial se composait donc de 113 entreprises, mais notre dénombrement final a été réduit à 75 pour diverses raisons, comme l'impossibilité de prendre contact avec l'entreprise ou le rejet des enquêtes erronées ou incomplètes.

Le profil des entreprises qui ont alimenté notre base de données est, comme nous l'avons déjà mentionné, considérablement différent de celui d'autres études nationales et internationales. L'effectif moyen de notre échantillon est de 118 travailleurs et le chiffre d'affaires, de 3067 millions de pesetas. En ce qui concerne la distribution sectorielle, nous remarquons la présence des activités les plus traditionnelles

2. Selon les banques de données consultées : IMPI, Fomento de la Producción, Dun and Bradstreet International, Dirnova'92 (IMPIVA), Economía3. 
de la base industrielle de la province d'Alicante qui se sont modelées sur les districts industriels : l'industrie du textile, de la chaussure, du marbre, des jouets et du nougat (Ybarra, 1992).

Le questionnaire utilisé lors de cette enquête se divise en sept blocs de variables. D'une part, nous avons les cinq blocs qui servent de base au classement (tableau 1) et, d'autre part, nous retrouvons deux autres blocs, dont la description complète se trouve à l'annexe, qui sont reliés à diverses politiques de production et au contexte de l'entreprise et qui permettront de faire ressortir les différences de comportement entre les groupes formés.

\section{Résultats}

Pour classer les entreprises étudiées, nous avons utilisé une série de facteurs qui résument les variables faisant partie des cinq groupes du tableau 1. Les facteurs ont été prélevés à l'aide d'une analyse des composantes principales, en réalisant une rotation orthogonale (VARIMAX) de la matrice factorielle afin de faciliter son interprétation et en prenant comme référence le niveau de $\pm 0,45$ de charge des variables dans les facteurs pour les attribuer à un facteur ou à un autre. De la même façon, nous avons éliminé toutes les variables dont la charge était supérieure à $\pm 0,45$ dans deux facteurs ou plus afin de garantir l'orthogonalité de ces derniers. Le nombre de facteurs retenus dans chaque cas a été décidé à l'aide de trois critères : les autovaleurs supérieures ou égales à l'unité, l'examen du graphique de sédimentation et le pourcentage de variabilité justifiée. Les facteurs obtenus avec chaque groupe de variables ainsi que la dénomination qui nous paraissait la plus adéquate pour ces derniers sont présentés au tableau 2.

Ensuite, nous avons réalisé une analyse de groupe à l'aide de l'algorithme de Ward, en utilisant la distance euclidienne au carré comme indice de similitude et les 15 facteurs définis auparavant comme variables. Pour décider du nombre de groupes à considérer, plusieurs critères ont été appliqués. Tout d'abord, nous avons essayé de suivre la recommandation de Lehmann (1979) et d'adapter le nombre de groupes à la dimension de la population $(n)$, de façon à situer ce nombre dans l'intervalle entre $n / 30$ et $n / 50$ pour limiter le nombre de groupes à deux ou trois. Par la suite, nous avons analysé l'accroissement de l'amplitude des groupes provoqué par cette réduction (Norusis, 1994) ainsi que la possibilité d'interpréter les résultats, ce qui nous a finalement donné trois groupes d'entreprises.

\section{Discussion}

\subsection{Caractérisation des trois groupes d'entreprises}

Les critères ayant servi à caractériser les trois groupes d'entreprises ont été les suivants : 
TABLEAU 2

Matrices factorielles (rotation VARIMAX)

\begin{tabular}{|c|c|c|c|}
\hline VARIABLES & & FACTEURS & \\
\hline \multicolumn{4}{|l|}{ ORIENTATION } \\
\hline FONCTIONNELLE & Transformatrice & Technologie & Marché \\
\hline Vente & 0,84459 & 0,04989 & 0,08423 \\
\hline Production & 0,79000 & 0,02195 & $-0,03142$ \\
\hline Finances & 0,76270 & 0,13147 & 0,01577 \\
\hline Design & $-0,06553$ & 0,83097 & 0,05755 \\
\hline Technologie & 0,23848 & 0,76474 & 0,02183 \\
\hline Commerciale & 0103688 & 0,06590 & 0,99569 \\
\hline DEMANDES & Différenciation & Temps & Prix \\
\hline Performances & 0,83973 & 0,01500 & $-0,26288$ \\
\hline Service après-vente & 0,67564 & $-0,00254$ & $-0,13943$ \\
\hline Qualité de conformité & 0,57771 & 0,10194 & 0,27233 \\
\hline Gamme & 0,57135 & 0,11676 & 0,25016 \\
\hline Ponctualité des livraisons & 0,08072 & 0,91386 & $-0,10677$ \\
\hline Rapidité des livraisons & 0,06293 & 0,88481 & 0,20017 \\
\hline Prix & $-0,01601$ & 0,04262 & 0,94295 \\
\hline FORCES & Temps & Différenciation & Prix \\
\hline Rapidité des livraisons & 0,97082 & 0,04820 & 0,00432 \\
\hline Ponctualité des livraisons & 0,95147 & 0,11213 & 0,08202 \\
\hline Performances & $-0,01233$ & 0,83200 & $-0,11999$ \\
\hline Gamme & 0,15546 & 0,74285 & 0,16481 \\
\hline Prix & 0,04479 & 0,02472 & 0,98557 \\
\hline OBJECTIFS DE PRODUCTION & Efficacité & Décongestion & Efficience \\
\hline Accomplir les programmes & 0,86914 & $-0,05167$ & 0,07027 \\
\hline Réduire les défauts & 0,86411 & 0,11416 & 0,04846 \\
\hline Accroître la flexibilité & 0,22840 & 0,85838 & $-0,08384$ \\
\hline Réduire les stocks & $-0,29674$ & 0,62302 & 0,36947 \\
\hline Réduire les coûts & 0,13196 & 0,04646 & 0,94095 \\
\hline \multicolumn{4}{|l|}{ SÉLECTION } \\
\hline DES FOURNISSEURS & Critères classiques & Proximité & Innovation \\
\hline Qualité & 0,85716 & 0,11337 & 0,07251 \\
\hline Prix & 0,81889 & $-0,14399$ & 0,06207 \\
\hline Ponctualité & 0,81300 & 0,24120 & 0,18129 \\
\hline Distance géographique & 0,07117 & 0,97882 & 0,04173 \\
\hline Capacité innovatrice & 0,14193 & 0,04279 & 0,98745 \\
\hline
\end{tabular}

- le classement des valeurs moyennes des variables d'origine intégrées à chaque ensemble de variables dans chacun des groupes (orientation fonctionnelle, demande des clients, assises de l'entreprise, objectifs de production et 
critères de sélection des fournisseurs). Ce classement apparaît entre parenthèses après la valeur moyenne de chaque variable dans les différents groupes (tableau 3);

- les différences au point de vue de l'évaluation moyenne de chaque variable dans les différents groupes. Pour vérifier l'importance statistique de ces différences, nous avons appliqué une analyse des écarts (ANOVA), qui contraste lorsqu'il existe des différences importantes dans les valeurs moyennes de chaque variable entre les trois groupes. Au tableau 3, nous avons marqué d'astérisques toutes les variables montrant des différences significatives pour un niveau inférieur à $0,1(*), 0,05\left(^{* *}\right)$ ou $0,01(* * *)$. Ensuite, afin d'identifier la ou les paires de groupes entre lesquels il existe des différences importantes, nous avons appliqué le test de Scheffe. Les résultats de ce dernier test sont résumés au tableau 3 , où l'on retrouve pour chaque variable, au besoin, la ou les paires de groupes entre parenthèses qui présentaient des différences significatives à un niveau inférieur à 0,05 lors des évaluations moyennes entre ces derniers ;

- les différences dans les ponctuations factorielles moyennes de chaque groupe (tableau 4), détectées avec l'analyse des écarts et le test de Scheffe. Dans ce cas et aussi en raison du processus de classification réalisé lors de l'analyse factorielle, il serait inutile d'étudier le classement des facteurs appartenant à un même groupe, les valeurs de ce dernier ne représentant pas son importance relative.

En partant de ces trois critères, nous obtenons trois groupes que nous pourrions appeler fabricants de produits haut de gamme, fabricants de produits de masse à court délai et fabricants de produits de masse orientés vers la variété. Nous allons maintenant décrire les caractéristiques de chaque groupe.

Le premier groupe comprend 29 firmes, représentant $39 \%$ de l'ensemble. La dénomination fabricants de produits haut de gamme présente les caractéristiques suivantes :

- ces fabricants ont une orientation fonctionnelle évidente vers le design du produit, car il figure en tête du classement des fonctions alors qu'il occupe le dernier rang dans les deux autres groupes ; l'évaluation moyenne de ces fabricants est nettement supérieure à celle du troisième groupe ;

- ils visent une clientèle qui exige un produit de haute qualité au regard de la conformité, de la performance et des délais de livraison, attributs qui occupent le premier rang du classement ; ce marché est prêt à payer des prix élevés, le prix occupant la cinquième place et présentant une valeur moyenne assez inférieure à celle du groupe 2 ; 
Tableau 3

Valeurs moyennes des variables dans les groupes

\begin{tabular}{|c|c|c|c|}
\hline & $n=29$ & $n=25$ & $n=21$ \\
\hline \multicolumn{4}{|l|}{ ORIENTATION FONCTIONNELLE } \\
\hline Achats*** $(12 ; 23)$ & $6,7586(6)$ & $8,6400(5)$ & $7,1429(3)$ \\
\hline Production $* * *(12 ; 23)$ & $7,8966(2)$ & $9,3200(\mathrm{l})$ & $8,1905(1)$ \\
\hline Finances*** $(12,23)$ & $7,1034(5)$ & $8,9200(3)$ & $6,8095(5)$ \\
\hline Design*** $(13,23)$ & $8,2759(1)$ & $7,9200(7)$ & $5,4762(7)$ \\
\hline Technologie*** $(13 ; 23)$ & $7,5172(4)$ & $8,5600(6)$ & $5,8095(6)$ \\
\hline Commerciale** (12) & $7,6897(3)$ & $9,0800(2)$ & $8,0000(2)$ \\
\hline Distribution $* * *(12 ; 23)$ & $6,4483(7)$ & $8,6800(4)$ & $7,0476(4)$ \\
\hline \multicolumn{4}{|l|}{ DEMANDES DES CLIENTS } \\
\hline Performance*** (13) & $8,2759(3)$ & $7,6400(5)$ & $6,1429(6)$ \\
\hline Service & $5,6552(7)$ & $4,9600(6)$ & $3,6190(7)$ \\
\hline Qualité de conformité & $8,7241(1)$ & $9,0800(3)$ & $7,9048(3)$ \\
\hline Gamme** (23) & $7,4828(6)$ & $8,0000(4)$ & $6,5238(5)$ \\
\hline Ponctualité des livraisons $* * *(12 ; 23)$ & $8,5862(2)$ & $9,4800(1)$ & $8,1905(2)$ \\
\hline Rapidité des livraisons*** $(12 ; 23)$ & $8,2069(4)$ & $9,3200(2)$ & $7,8571(4)$ \\
\hline Prix*** (12) & $7,5517(5)$ & $9,3200(2)$ & 8,6667 (l) \\
\hline \multicolumn{4}{|l|}{ FORCES DE L'ENTREPRISE } \\
\hline Rapidité des livraisons*** $(13 ; 23)$ & $7,1034(5)$ & $7,4400(2)$ & $5,5238(4)$ \\
\hline Ponctualité des livraisons*** $(13 ; 23)$ & $7,1734(4)$ & $7,7600(1)$ & $5,2857(5)$ \\
\hline Performance ${ }^{* * *}(12 ; 13 ; 23)$ & $8,0000(1)$ & $6,8400(4)$ & $5,7143(3)$ \\
\hline Gamme & $7,2069(3)$ & $6,4000(5)$ & $6,3333(1)$ \\
\hline Prix*** $(12 ; 23)$ & $4,0000(7)$ & $6,3200(7)$ & $5,0000(7)$ \\
\hline Qualité de conformité*** (13) & $7,5517(2)$ & $6,9600(3)$ & $5,9048(2)$ \\
\hline Service après-vente $* * *(13 ; 23)$ & $6,4138(6)$ & $6,3600(6)$ & $5,0952(6)$ \\
\hline \multicolumn{4}{|l|}{ OBJECTIFS DE PRODUCTION } \\
\hline Accomplir les programmes** (12) & $7,7931(3)$ & $9,3600(1)$ & $8,1905(2)$ \\
\hline Réduire les défauts** (12) & $7,9310(2)$ & $9,2000(2)$ & $8,2857(1)$ \\
\hline Accroître la flexibilité & $6,8276(5)$ & $7,8400(3)$ & $7,7619(3)$ \\
\hline Réduire les stocks & $7,3103(4)$ & $7,3200(5)$ & $6,8095(6)$ \\
\hline Réduire les coûts*** $(12 ; 23)$ & $8,1724(1)$ & $9,3600(1)$ & $7,5238(4)$ \\
\hline Réduire le temps & $6,7931(6)$ & $7,6000(4)$ & $6,9524(5)$ \\
\hline \multicolumn{4}{|l|}{ SÉLECTION DES FOURNISSEURS } \\
\hline Qualité & $8,8966(1)$ & $9,4400(1)$ & $9,3810(1)$ \\
\hline Prix** (12) & $7,9655(3)$ & $9,1600(2)$ & $8,3333(3)$ \\
\hline Ponctualité & $8,4138(2)$ & $9,1200(3)$ & $8,4286(2)$ \\
\hline Distance géographique & $3,4483(6)$ & $3,7200(6)$ & $4,3333(5)$ \\
\hline Capacité innovatrice ${ }^{* * *}(12 ; 13 ; 23)$ & $6,0345(5)$ & $8,2000(5)$ & $3,8571(6)$ \\
\hline Temps de livraison & $7,5172(4)$ & $8,5600(4)$ & $7,9048(4)$ \\
\hline
\end{tabular}




\section{TABLEAU 4}

Valeurs moyennes des facteurs dans les trois groupes

\begin{tabular}{lrrr}
\hline & $n=29$ & $n=25$ & $n=21$ \\
\hline ORIENTATION FONCTIONNELLE & & & \\
Transformatrice*** $(12 ; 23)$ & $-0,4860$ & 0,7882 & $-0,2672$ \\
Technologique*** $(13 ; 23)$ & 0,3087 & 0,3492 & $-0,8420$ \\
Vers le marché** $(12)$ & $-0,2895$ & 0,3834 & $-0,0566$ \\
\hline DEMANDES DES CLIENTS & & & \\
Différenciation*** $(13 ; 23)$ & 0,2868 & 0,1939 & $-0,6269$ \\
Temps*** $(12 ; 23)$ & $-0,1896$ & 0,5838 & $-0,4455$ \\
Prix*** $(12)$ & $-0,4418$ & 0,4391 & 0,0874 \\
\hline FORCES DE L'ENTREPRISE & & & \\
Temps *** $(13 ; 23)$ & 0,1530 & 0,4078 & $-0,6968$ \\
Différenciation *** (12; 13) & 0,5694 & $-0,1942$ & 0,5552 \\
Prix *** $(12 ; 13)$ & $-0,5830$ & 0,6000 & 0,0909 \\
\hline OBJECTIFS DE PRODUCTION & & & \\
Efficacité $* * *(12)$ & $-0,3725$ & 0,4996 & $-0,0803$ \\
Décongestion & $-0,1533$ & 0,0889 & 0,1059 \\
Efficience *** (23) & $-0,0453$ & 0,5161 & $-0,5519$ \\
\hline SÉLECTION DES FOURNISSEURS & & & \\
Critères classiques & $-0,2690$ & 0,2724 & 0,0473 \\
Proximité & $-0,0496$ & $-0,1092$ & 0,1984 \\
Capacité innovatrice*** $(12 ; 13 ; 23)$ & 0,0143 & 0,6250 & $-0,7638$ \\
\hline
\end{tabular}

- l'avantage compétitif de ces fabricants repose sur l'offre d'un produit de haute performance (première place au classement et valeurs supérieures aux groupes 2 et 3 ), ce qui leur permet de demander des prix supérieurs à ceux de la concurrence; c'est le groupe qui possède la plus petite valeur dans ce champ de compétences et les différences avec le groupe 2 et le groupe 3 sont assez importantes, si l'on considère le facteur de différenciation au tableau 4 ;

- les priorités de la gestion de l'entreprise sont orientées vers la réduction des coûts (en première position) et la recherche de la flexibilité n'est pas une priorité (cinquième position; elle occupe la troisième dans les groupes 2 et 3), probablement parce que le processus est, par nature, assez adaptable ;

- le manque d'intérêt de la part des clients en ce qui concerne le prix est aussi ressenti par les fournisseurs, ce qui rend ces entreprises moins exigeantes que celles du groupe 2 au regard des prix des fournisseurs. 
Le deuxième groupe, fabricants de produits de masse à court délai, comprend 25 entreprises et représente $33 \%$ de l'échantillon. Les principales caractéristiques de ce groupe, en prenant en considération les données des tableaux 3 et 4 , sont les suivantes :

- bien que ces fabricants reconnaissent la grande importance de la technologie et du design (sa valeur moyenne est comparable à celle du groupe 1 et supérieure à celle du groupe 3 ), ils considèrent que les autres fonctions sont plus critiques encore pour leur compétitivité, car les deux précédentes occupent les dernières positions au classement ;

- ces fabricants exercent leur activité dans un marché très exigeant au point de vue du prix, de la rapidité et de la ponctualité des livraisons, car ces attributs se situent au premier rang du classement et les deux derniers aspects dépassent nettement ceux des groupes 1 et 2 ;

- les entreprises de ce groupe ont obtenu la plus grande force au niveau des prix, ce qui correspond évidemment aux fabricants de produits de masse (avec des différences significatives par rapport aux deux autres groupes), bien que leur plus grande force face à la concurrence ne réside pas dans ce dernier aspect, mais plutôt dans l'excellence de leur temps de réponse (la rapidité et la ponctualité occupent les premières positions au classement des forces);

- ces fabricants recherchent intensivement la façon d'améliorer les fondements de leur avantage compétitif (coûts et livraison) dans la gestion de l'entreprise, car l'accomplissement des contrats et la réduction des coûts partagent la première place dans les priorités de gestion de l'entreprise, avec certaines différences importantes par rapport au groupe 1 et au groupe 3 pour ce qui est de la réduction des coûts;

- ces entreprises semblent confier le problème de l'innovation à leurs fournisseurs - peut-être pour mieux centrer leurs efforts sur le rendement des processus internes - et se montrent assez exigeants au point de vue des prix. C'est pourquoi l'évaluation de la capacité d'innovation des fournisseurs est nettement supérieure à celle des autres groupes et le prix se situe en deuxième place, avec des valeurs supérieures à celles du groupe 1 .

Le troisième groupe, qui représente $28 \%$ des entreprises étudiées, comprend 21 firmes. Nous avons convenu de les appeler fabricants de produits de masse orientés vers la variété en raison des particularités suivantes :

- comme les entreprises du groupe 2, ces fabricants ont relégué le design et la technologie aux dernières places du classement des fonctions, mais, pour eux, l'évaluation de ces fonctions est moins importante que pour le 
reste des entreprises, signe que ces fabricants utilisent un processus productif combiné à une technologie de pointe et que leur produit subit peu d'innovation au point de vue du design;

- ils offrent des produits moins performants (sixième place au classement des demandes) dans un marché très exigeant au niveau du prix (première place), ce qui fait d'eux des fabricants de masse, tout comme ceux du groupe 2 ;

- les forces développées par ces entreprises ne semblent pas s'adapter aux demandes des clients, car leur capacité d'offrir des prix très bas occupe tout de même la dernière place du classement, en plus d'être nettement inférieure à celle du groupe 2 (que nous avons aussi qualifié de fabricants de masse) et de dépasser à peine celle du groupe 1. Paradoxalement, la variété des produits occupe la première place au classement des forces alors qu'elle occupait la cinquième dans les préférences des clients. Cela nous amène à considérer ce groupe, qui semble opérer sur un marché de masse, comme étant réorienté vers la variété, puisque ces entreprises semblent compenser leur faiblesse au niveau des prix en offrant une gamme de produits plus étendue ;

- en conséquence, la gestion de l'entreprise vise plutôt l'amélioration de la flexibilité que la réduction des coûts. Ainsi, on remarque que ce groupe est le seul à considérer la flexibilité comme étant prioritaire (troisième place) devant la réduction des coûts (quatrième place, alors qu'elle occupe la première dans les groupes 1 et 2);

- l'avancement technologique du produit ainsi que le processus semblent devenir la responsabilité des fournisseurs, puisque la capacité d'innovation de ces derniers demeure un critère très secondaire lors de leur sélection (avec un pointage nettement inférieur à celui des autres groupes), malgré l'importance de la fonction d'achat (qui, de la sixième et cinquième place des groupes 1 et 2 , passe à la troisième).

\subsection{Analyse discriminante}

Afin de valider les résultats du groupe d'analyse qui vient d'être commenté, nous réalisons une analyse discriminante qui nous servira aussi à caractériser les trois groupes, identifiés à partir de l'étude de la distribution des groupes, en fonctions canoniques discriminantes.

Étant donné la grande quantité de variables utilisées pour effectuer le classement du groupe d'analyse, nous avons décidé de réaliser l'analyse discriminante avec la méthode stepwise qui permet d'inclure uniquement les principales variables pour diviser l'échantillon en différents groupes. Le critère appliqué pour la sélection 
des variables a été la réduction du Lambda de Wilks, en établissant un niveau de 3,84 pour l'entrée d'une nouvelle variable et de 2,71 pour la sortie. De cette façon, les résultats obtenus ne sont basés que sur 9 des 33 variables d'origine.

Le tableau 5 présente les principaux résultats de l'analyse discriminante. Nous avons obtenu une valeur de 0,14 pour le Lambda de Wilks avec un niveau de signification inférieur à 0,0001 , ce qui nous permet de réfuter l'hypothèse voulant que la moyenne des deux fonctions canoniques soit égale dans les trois groupes considérés. En outre, chacune des deux fonctions canoniques, qui définissent les orientations stratégiques des entreprises analysées, présente une corrélation élevée et positive, tout comme les autovaleurs ; ce qui nous indique que ces deux fonctions canoniques permettront de mieux déterminer les groupes différents parmi ceux proposés. Enfin, le fait que les pourcentages de variabilité expliquée pour chacune des fonctions soient assez élevés en termes relatifs $(55,13 \%$ et $44,87 \%)$ signale que ces deux fonctions contribuent à différencier les groupes.

TABLEAU 5

Résultats de l'analyse discriminante

\begin{tabular}{ccccc}
\hline Fonction & Autovaleur & $\begin{array}{c}\text { Variabilité } \\
\text { \% }\end{array}$ & $\begin{array}{c}\text { Variance accumulée } \\
\text { \% }\end{array}$ & $\begin{array}{c}\text { Corrélation } \\
\text { canonique }\end{array}$ \\
\hline 1 & 1,85094 & 55,13 & 55,13 & 0,8057533 \\
2 & 1,50632 & 44,87 & 100 & 0,7752476 \\
\hline
\end{tabular}

\begin{tabular}{lrrrr}
\hline Variable & Fonction 1 & Fonction 2 & Fonction 1 & Fonction 2 \\
\hline Force en prix & 0,43050 & 0,06167 & 0,68634 & $-0,14275$ \\
Fonction d'achats & 0,39129 & 0,16456 & 0,52237 & $-0,11797$ \\
Réduction des défauts à l'usine & 0,25162 & 0,08230 & 0,42951 & $-0,38002$ \\
Qualité du fournisseur & 0,13043 & $-0,04985$ & 0,40964 & $-0,29790$ \\
\hline Force en ponctualité & 0,07000 & 0,49197 & $-0,19185$ & 0,59375 \\
Capacité d'innovation & & & & \\
du fournisseur & 0,21874 & 0,47046 & 0,21716 & 0,66686 \\
Force en performances & $-0,27835$ & 0,40311 & $-0,72152$ & 0,46383 \\
Réduction des coûts à l'usine & 0,21866 & 0,31455 & 0,18997 & 0,45945 \\
Demandes de ponctualité & 0,21942 & 0,28265 & 0,28342 & 0,37964 \\
\hline
\end{tabular}

L'interprétation des fonctions discriminantes est réalisée à partir des coefficients standardisés des fonctions en question et des corrélations des variables avec les fonctions, coefficients qui sont présentés au tableau 5 en ordre décroissant.

D'après les données obtenues, nous pouvons appeler la première fonction producteur efficient, puisque la principale corrélation est représentée par la force 
au niveau des prix et que les coefficients standardisés avec plus de poids sont, à nouveau, la force des prix (positive) et celle de la performance (négative).

Nous avons appelé la deuxième fonction création de valeur pour le client, étant donné les grandes charges et corrélations qu'elle présente pour deux forces de l'entreprise qui permettent d'augmenter, d'une part, la valeur du produit pour le client (performance et ponctualité des livraisons) et, d'autre part, la capacité innovatrice du fournisseur, cela entraînant une dynamique de création de produits de meilleure valeur, même pour le client.

La distribution des entreprises de chacun des trois groupes dans les axes correspondants aux deux fonctions discriminantes apparaît à la figure 1, de même que le centre de gravitation (centroïde) de chaque groupe.

FIGURE 1

Fonctions canoniques discriminantes

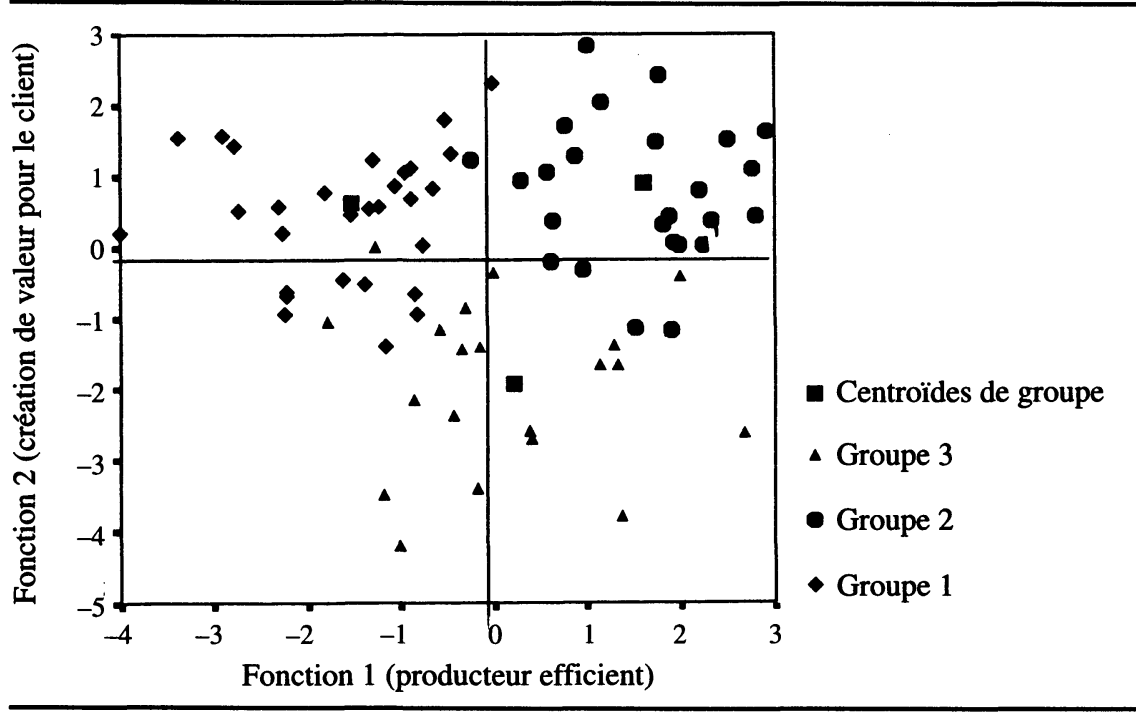

À la figure 1, nous voyons que les entreprises du premier groupe ont obtenu un résultat élevé dans la deuxième fonction discriminante. Il s'agit donc de fabricants capables de créer un produit de grande valeur pour le client puisque, comme nous l'avons vu, sa principale force réside dans les performances du produit. Les fabricants de produits haut de gamme, comme nous avons appelé les fabricants du premier groupe, offrent un produit plus cher et, par conséquent, se situent dans la partie gauche du graphique avec des valeurs réduites dans la première fonction discriminante (producteur efficient). 
Les entreprises du deuxième groupe occupent une place semblable à celles du premier par rapport à l'axe vertical (deuxième fonction discriminante) bien qu'elle soit un peu décalée vers la droite, avec de fortes valeurs, également, dans la première fonction discriminante. Il s'agit donc de fabricants très efficaces et capables de créer un produit ayant une forte valeur ajoutée pour le client. Enfin, ces entreprises, que nous avons appelées fabricants de produits de masse à court délai, arrivent à s'occuper efficacement de leurs clients (caractéristique des fabricants de masse), en offrant en plus un produit possédant les attributs que ce genre de consommateurs préfère (ponctualité dans les livraisons, dans ce cas).

Finalement, les entreprises du troisième groupe (fabricants de produits de masse orientés vers la variété) présentent un résultat moyen dans la première fonction discriminante (producteur efficient) ainsi que des valeurs très faibles dans la seconde (création de valeur pour le client). Les entreprises de ce groupe ne semblent pas être aussi efficaces que celles du deuxième groupe, car elles tentent d'offrir une certaine variété de produits, sans toutefois arriver à créer une grande valeur ajoutée pour le client, puisqu'elles ciblent en fait un marché de masse où le prix a plus d'importance que la variété.

Enfin, nous signalerons un autre élément à considérer pour mesurer la validité du classement effectué avec le groupe d'analyse. Il s'agit de la matrice de confusion du tableau 6 , où nous comparons les classements effectués avec l'analyse discriminante et le groupe d'analyse. Nous pouvons constater que l'analyse discriminante d'origine classe correctement $89,33 \%$ des entreprises, plus précisément $89,7 \%$ des entreprises du groupe $1,92 \%$ du groupe 2 et $85,7 \%$ du groupe 3 . La validation croisée, avec laquelle chaque cas se classe moyennant les fonctions dérivées à partir du reste des cas, a donc classé correctement $81,3 \%$ des entreprises, ainsi que $82,8 \%$, $88 \%$ et $71,4 \%$ des cas en ce qui concerne les entreprises des groupes 1,2 et 3 respectivement.

TABleaU 6

Résultats du classement

\begin{tabular}{lrrrr}
\hline Original & \multicolumn{4}{c}{ Groupe pronostiqué } \\
\hline Groupe & $n$ & 1 & \multicolumn{1}{c}{2} & \multicolumn{1}{c}{3} \\
\hline 1 & 29 & $26(89,7 \%)$ & $1(3,4 \%)$ & $2(6,9 \%)$ \\
2 & 25 & $1(4 \%)$ & $23(92 \%)$ & $1(4 \%)$ \\
3 & 21 & $2(9,5 \%$ & $1(4,8 \%)$ & $18(85,7 \%)$ \\
\hline Validation croisée & \multicolumn{5}{c}{} \\
\hline 1 & 29 & $24(82,2 \%)$ & $2(6,9 \%)$ & $3(10,3 \%)$ \\
2 & 25 & $1(4 \%)$ & $22(88 \%)$ & $2(8 \%)$ \\
3 & 21 & $4(19 \%)$ & $2(9,5 \%)$ & $15(71,4 \%)$ \\
\hline
\end{tabular}




\subsection{Politiques de production}

Après avoir réalisé et validé le classement des entreprises dans les trois groupes stratégiques, nous allons étudier le développement de certaines politiques dans chacun des groupes en examinant le pourcentage des entreprises ayant déclaré, dans chaque groupe, déjà appliquer une série de pratiques et de politiques de production (voir annexe). Pour analyser la validité statistique des différences entre les groupes, nous avons utilisé la méthode du $\chi^{2}$ et obtenu un total de trois résultats différents pour chaque variable. Ainsi, nous avons représenté à la figure 2 les pourcentages des cas dans lesquels on applique les techniques ou les politiques énumérées, en indiquant le ou les groupes dont les chiffres sont significativement différents (supérieurs ou inférieurs), avec un niveau de signification de $5 \%$ par rapport au reste des entreprises. Notons que le rapport entre les politiques de production n'inclut pas les entreprises pour lesquelles nous n'avons pas trouvé de différences significatives.

FIGURE 2

Politiques de production

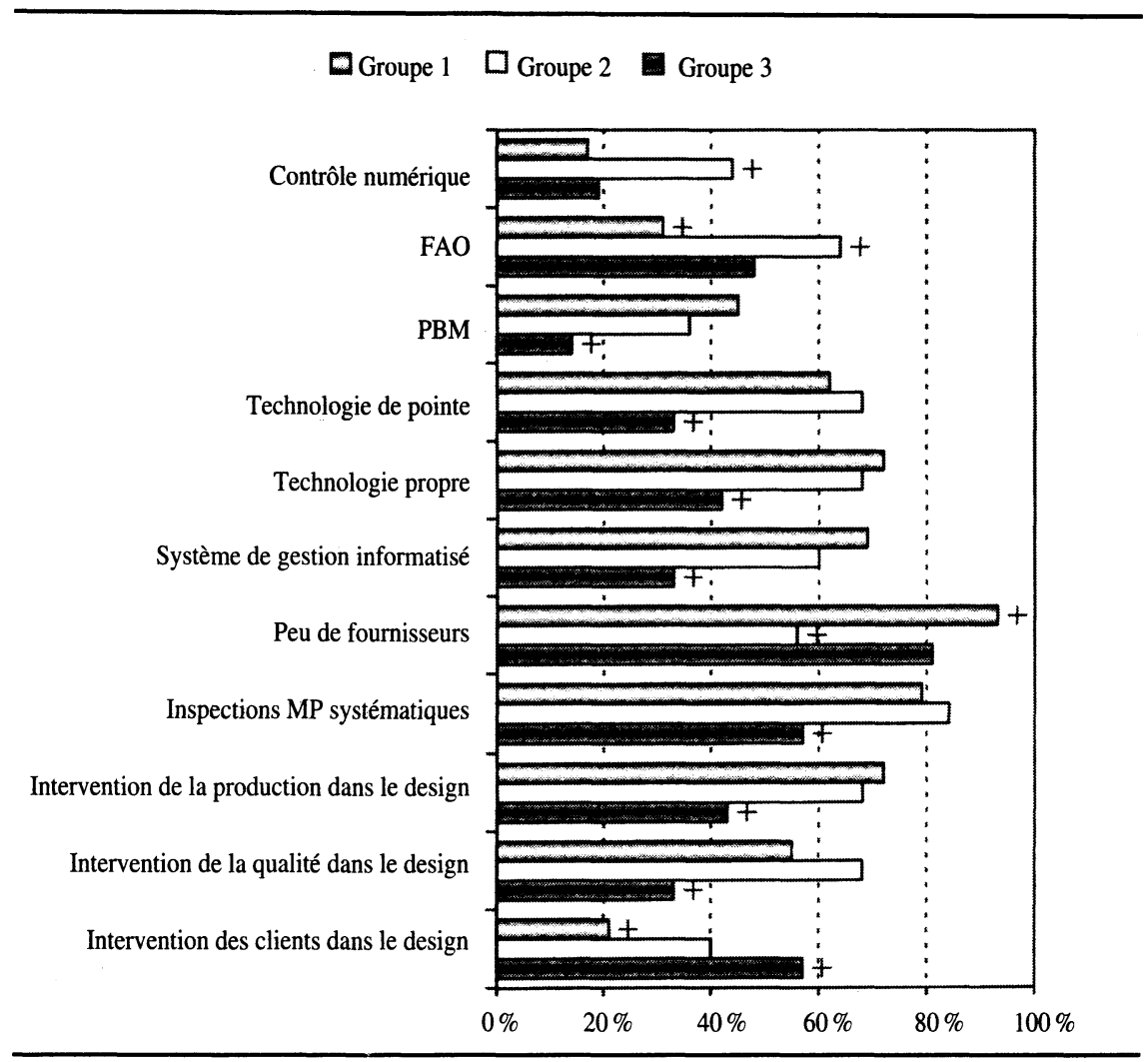


À la figure 2, nous pouvons constater que plus de la moitié des 11 politiques de production présentant des différences significatives, soit les 6 premières de la liste, sont reliées à la technologie de production, que 2 ont un lien avec la gestion des fournisseurs et 3 , avec les groupes ayant participé au design du produit.

Les différences relevées dans le groupe de variables reliées à la technologie confirment la faible orientation des entreprises du groupe 3 vers celle-ci; fait que nous avions déjà remarqué lors de l'analyse de l'importance des différentes fonctions entrepreneuriales. Ces entreprises ont affirmé avoir utilisé des systèmes de gestion très informatisés ainsi que des systèmes de planification (PBM) et avoir développé une technologie propre et de pointe dans beaucoup moins de cas que les autres entreprises. En ce qui concerne le contrôle numérique et les systèmes FAO, nous pouvons affirmer que les entreprises du deuxième groupe sont celles qui intègrent le plus souvent ces technologies à leurs systèmes de production.

Pour ce qui est de la gestion des fournisseurs, les résultats montrent que les entreprises du deuxième groupe sont celles qui favorisent le plus la concurrence entre leurs fournisseurs pour tenter, semble-t-il, de profiter de l'innovation et même de stimuler cette dernière dans le marché des matières premières, car elle a une grande valeur aux yeux des fabricants de produits de masse à court délai, comme nous l'avons vu dans les caractéristiques des entreprises de ce groupe. En outre, les entreprises du troisième groupe sont celles qui ont le moins souvent déclaré effectuer des contrôles systématiques de la qualité des matières premières. À notre avis, cela est relié au fait que ce groupe d'entreprises présente une plus grande homogénéité dans le domaine des matières premières (il s'agit d'un produit mature et la capacité d'innovation du fournisseur est peu valorisée); il est donc plus facile d'employer des méthodes d'échantillonnage et même de permettre le «libre passage » de la marchandise.

Les trois dernières variables (figure 2) représentent le pourcentage de cas, dans chaque groupe, où l'on relève la participation d'employés des secteurs de la production, du contrôle de la qualité, ainsi que celle du client dans la conception du produit. Les résultats indiquent qu'il existe chez les fabricants de produits de masse orientés vers la variété (troisième groupe) une coopération interfonctionnelle (entre production et qualité) plus faible dans la création de nouveaux designs que chez les deux autres groupes, bien que la participation du client soit plus grande. Ainsi, la volonté de ces entreprises de varier leur offre afin de compenser la faiblesse de leurs prix fait en sorte que le client peut décider de certains aspects du design du produit, en dépit de la maturité technologique de ce dernier ; étant donné la stabilité du produit, le design ne constitue plus un problème, sa résolution ayant même permis d'éclaircir certaines interrogations relatives à la production et à la qualité. Cette situation contraste avec celle des fabricants de produits haut de gamme (premier groupe) pour lesquels la technologie du produit permet très rarement une adaptation au goût de chaque client, même si ce produit est destiné au secteur le plus raffiné du marché. 


\subsection{Contexte stratégique}

La dernière étape de notre recherche porte sur l'étude de certaines variables du contexte stratégique des entreprises dans les trois groupes, où nous avons tenté de relever des différences significatives. Pour ce faire, nous avons prélevé au tableau 7 toutes les variables présentant des différences significatives selon l'analyse des écarts (ANOVA), ainsi que les groupes entre lesquels ces différences étaient significatives à un niveau inférieur à 0,05 .

TABleau 7

Variables du contexte stratégique

\begin{tabular}{lccc}
\hline Variable & $\begin{array}{c}\text { Groupe 1 } \\
(\boldsymbol{n}=\mathbf{2 9})\end{array}$ & $\begin{array}{c}\text { Groupe 2 } \\
(\boldsymbol{n}=25)\end{array}$ & $\begin{array}{c}\text { Groupe 3 } \\
(\boldsymbol{n}=\mathbf{2 1})\end{array}$ \\
\hline Exportation ** $(13)$ & 3,2069 & 2,8400 & 2,1905 \\
Utilisation de la capacité * & 2,4138 & 3,0800 & 3,2857 \\
Temps de réception MP ** $(13)$ & 3,3793 & 3,1200 & 2,3810 \\
Réserve de MP *** $(13 ; 23)$ & 2,4828 & 2,3200 & 1,9048 \\
\hline
\end{tabular}

Dans ce tableau, on remarque que les différences entre les groupes ne concernent que l'orientation exportatrice, l'utilisation de la capacité, le temps de réponse du fournisseur et la réserve minimale des matières premières. Par contre, les mesures de rendement de l'unité (évolution de la rentabilité de l'entreprise, de sa compétitivité et du fonctionnement général de l'usine au cours des quatre dernières années), les autres mesures du rendement au point de vue logistique, le processus de production utilisé, les mesures de volume de l'entité ainsi que le secteur d'activité n'ont pas donné de résultats significatifs lors de l'application du test correspondant.

Si nous analysons les variables présentant des différences statistiques significatives, nous remarquons que les entreprises du groupe 1 ont tendance à exporter une plus grande part de leur chiffre d'affaires que celles du groupe 3 , ce qui indique que parmi les grandes entreprises de la province d'Alicante, les fabricants de produits haut de gamme exportent plus que les fabricants de produits de masse orientés vers la variété.

Les données du tableau 7 montrent qu'il existe des différences entre les entreprises des trois groupes à un niveau inférieur à $10 \%$ au regard de l'utilisation de la capacité. Cependant, le test de Scheffe n'a pas donné de résultats significatifs, même si les valeurs moyennes semblaient indiquer que les entreprises des groupes 2 et 3 utilisaient plus leur capacité que celles du groupe 1 . Ces différences concordent avec le fait que les entreprises du premier groupe (fabricants de produits haut de gamme) sont censées être, par nature, moins efficaces dans l'exploitation de leur 
capacité de production que les fabricants de masse (groupes 2 et 3 ); les résultats permettent de traiter de cette question presque uniquement à titre indicatif sans justification au point de vue statistique.

Selon les deux mesures du rendement logistique, les entreprises du troisième groupe (fabricants de produits de masse orientés vers la variétê) présentent un meilleur synchronisme avec leurs fournisseurs. En effet, le temps de réponse de leurs fournisseurs est nettement plus court que celui des fournisseurs des entreprises du groupe 1 et les matières premières demeurent beaucoup moins longtemps dans l'entrepôt que celles des entreprises des groupes 1 et 2 . Nous pouvons attribuer ce comportement des entreprises du troisième groupe à l'homogénéité de la matière première utilisée (en comparaison des entreprises des groupes 1 , elles fabriquent un produit plus mature, et de celles du groupe 2 , il y a moins de changement dans le jeu des facteurs) ou encore à une tentative de transférer le coût de l'entretien des matières premières, en amont, aux fournisseurs.

\subsection{Comparaison avec d'autres travaux}

En principe, les particularités des entreprises de notre échantillon laissaient entrevoir peu de possibilités de comparaison avec d'autres travaux taxinomiques; un examen a posteriori des résultats obtenus dans le classement a confirmé cette crainte. Cependant, nous allons exposer, dans les lignes qui suivent, les parallèles établis avec certains classements issus d'autres travaux.

Parmi les trois groupes identifiés par Miller et Roth (1994) - conservateurs (caretakers), commerciaux (marketeers) et innovateurs (innovators) - seuls les innovateurs présentent une certaine ressemblance avec nos fabricants de produits haut de gamme, car tous les deux valorisent le design du produit et placent la qualité conformité et la performance en tête de leurs priorités. Par contre, les conservateurs basent leur compétitivité exclusivement sur la fabrication de produits bon marché et les commerciaux, sur la rapidité de production et l'étendue de leur gamme de produits, ce qui n'équivaut aux caractéristiques d'aucun des groupes de notre étude.

Le double classement effectué par De Meyer (1992), basé sur des données recueillies en 1987 et 1988, dégage un groupe de fabricants de produits haut de gamme (high-performance product group) possédant la même dénomination que notre premier groupe ainsi que des caractéristiques semblables, par exemple l'accent mis sur les performances du produit et sur sa conformité. Les fabricants orientés vers le marketing (marketing oriented) identifiés par De Meyer correspondent à notre deuxième groupe (fabricants de produits de masse à court délai) au niveau de la rapidité de livraison et de la conformité comme forces importantes, reléguant la performance au deuxième rang. Finalement, les fabricants innovateurs (manufacturing innovators) identifiés par le même auteur ne présentent aucun lien 
avec notre dernier groupe (fabricants de produits de masse orientés vers la variété) mais possèdent des caractéristiques communes avec nos deux premiers au niveau de la livraison.

Avec le classement de Sweeney et Szwejczewsky (1996), il est relativement simple d'établir des liens avec les trois catégories que nous proposons. Premièrement, leurs fabricants innovateurs (innovators) se distinguent par la qualité et la performance tout comme ceux de notre premier groupe. Deuxièmement, ces auteurs ont un groupe d'entreprises appelé réorganisateurs (reorganizers) qui, comme les entreprises de notre deuxième groupe, mettent l'accent sur la rapidité et la fiabilité des livraisons. Troisièmement, les commerciaux (marketeers) partagent avec nos fabricants de produits de masse orientés vers la variété la force en ce qui concerne la conformité et la performance (ces auteurs ne prennent pas la gamme en considération), forces qui occupent les premières places au classement, sans toutefois accéder aux niveaux atteints dans ces dimensions par les innovateurs.

Finalement, les résultats de l'étude portant sur les grandes entreprises industrielles espagnoles réalisée par Avella, Fernández et Vázquez (1996) nous offrent une taxinomie (fabricants flexibles axés sur le marché, fabricants de produits de qualité à prix réduits et fabricants axés sur les livraisons) n'ayant de lien avec notre classement que dans une seule catégorie. Ainsi, les fabricants de produits à prix réduit ont en commun avec nos fabricants de produits haut de gamme la supériorité dans certaines dimensions de la qualité (produits durables sans imperfections et satisfaction du client). En ce qui concerne les deux autres groupes de fabricants, nous ne trouvons pas d'équivalence parfaite avec ceux de notre classement, car dans les deux cas, la force dans le domaine du temps se combine avec d'autres dimensions, mais cela nous permet toutefois d'associer ces deux groupes à nos fabricants de produits de masse à court délai.

\section{Conclusion}

Dans cet article, nous avons réalisé un classement des stratégies de production de 75 PME de la province d'Alicante en Espagne, en prenant comme point de repère la méthodologie d'autres études et en l'adaptant aux particularités de notre échantillon. Les résultats montrent qu'il existe trois groupes d'entreprises qui se distinguent par leur stratégie de production. Ce classement sert à mettre en relief les compétences à développer dans le secteur de la production selon les critères de compétitivité privilégiés.

Ainsi, les entreprises du premier groupe (fabricants de produits haut de gamme) optent pour une stratégie de différenciation en offrant des produits chers et de haute performance et en satisfaisant les demandes de leurs clients. Pour ces entreprises, le design et la technologie sont des aspects très importants à considérer 
pour accroître leur avantage compétitif et leur gestion doit viser la réduction des coûts plutôt que l'amélioration de la flexibilité, car les procédés de production sont intrinsèquement flexibles.

Les fabricants de produits de masse à court délai parviennent à satisfaire adéquatement les besoins d'un marché très exigeant au niveau des prix et des livraisons. Pour cela, la gestion de l'usine doit être orientée de façon à contribuer activement à développer ces caractéristiques, en mettant l'accent sur la réduction des coûts et la réalisation des tâches. Dans la sélection et l'évaluation des fournisseurs, ces entreprises insistent particulièrement sur la capacité d'innovation ainsi que sur les prix, en essayant de conserver ces caractéristiques en faisant affaire avec un grand nombre de fournisseurs pouvant, le cas échéant, se concurrencer.

Finalement, les fabricants de produits de masse orientés vers la variété se dirigent, semble-t-il, avec un produit mature, vers un marché de masse très exigeant au regard des prix, tout en essayant de compenser leur faiblesse dans ce domaine - qui peut être due en partie à l'utilisation d'une technologie de production de base en offrant une gamme plus étendue de produits. Cela se répercute sur la gestion de l'usine où la flexibilité prime sur la réduction des coûts.

Il faut rappeler qu'il s'agit ici de pratiques qui ont effectivement cours dans les entreprises étudiées, mais que nous ne pouvons pas déduire que ces pratiques sont les meilleures malgré leur utilisation dans ces entreprises. Le caractère normatif des résultats présentés devra toujours être considéré en tenant compte de la remarque précédente. C'est pour cette raison que nous exposerons, dans les paragraphes qui suivent, les limites de cette étude tout en faisant quelques recommandations pour de futures recherches.

Tout d'abord, nous n'avons pas relevé de différences de rendement entre les trois groupes d'entreprises; en d'autres termes, il n'y a pas de stratégie préférable à une autre, qui permettrait aux entreprises d'atteindre de meilleurs résultats. Cependant, il serait intéressant d'étudier cette question avec un plus grand échantillon, en prenant en considération d'autres variables comme le secteur industriel, afin de vérifier si certaines stratégies procurent de meilleurs ou de pires résultats que d'autres.

De plus, il serait intéressant d'approfondir l'étude de chacun des groupes, en utilisant cette fois encore un plus grand échantillon, afin d'identifier les caractéristiques des entreprises obtenant les meilleurs et les pires résultats dans chaque groupe. En définitive, les futures recherches devraient examiner les relations de causalité entre la stratégie de fabrication, les politiques et les pratiques de production ainsi que les résultats.

Enfin, nous devons parler de l'échantillon utilisé. Tout d'abord, ii faut rappeler que cet échantillon se limite à un territoire géographique déterminé (la province 
d'Alicante) et que, par conséquent, les résultats ne sont pas nécessairement applicables à d'autres territoires plus vastes. Ensuite, nous devons signaler certaines particularités du secteur industriel d'Alicante, dont le fait que la sous-traitance est une pratique très courante ; en effet, $70 \%$ des entreprises étudiées ont recours à cette méthode. Dans les recherches ultérieures, on devrait vérifier si les stratégies de production des entreprises sous-traitantes ont un lien étroit avec celles de leurs clients et, plus spécialement, si l'on relève une stratégie commune entre les entreprises intégrées aux réseaux commerciaux.

Pour finir, nous devons signaler certaines limites dans la méthodologie employée. Ainsi, les variables utilisées font uniquement état des perceptions de la direction qui ne coïncident pas nécessairement avec les mesures objectives du phénomène correspondant (Tosi, Aldag et Storey, 1973). Cependant, il faut souligner, d'une part, que ces variables sont fréquemment utilisées dans les recherches sur les organisations et que leur utilisation a été justifiée par plusieurs auteurs dont Lawrence et Lorsch (1967) ou Powell (1992) et que certains auteurs, comme Reger et Huff (1993) ou Powell (1996) affirment que les perceptions peuvent être même plus significatives que les mesures objectives lorsqu'il s'agit d'évaluer la stratégie de l'entreprise. Il serait possible de résoudre une partie de ce problème en ayant recours aux diverses sources d'information fournies par les entreprises, par exemple en communiquant avec deux ou trois gestionnaires de chaque entreprise ou encore en combinant les variables objectives avec les subjectives.

\section{Bibliographie}

Avella, L., E. FernándeZ et C.J. VÁzQueZ (1996), « Clasificación de las estrategias de fabricación de las grandes empresas industriales españolas », Economía Industrial, $n^{0} 311$, p. 77-92.

BAMBERGER, I. (1989), «Developing competitive advantage in small and medium-size firms ", Long Range Planning, vol. 22, no 5, p. 80-88.

BeCATtini, G. (1989), «Los distritos industriales y el reciente desarrollo italiano », Sociología del Trabajo, $\mathrm{n}^{\circ}$ 5, p. 3-17.

Beckman, S.L., W.A. Boller, S.A. Hamilton et J.W. Monroe (1990), «Using manufacturing as a competitive weapon: the development of a manufacturing strategy », dans P.E. Moody (dir.), Strategic Manufacturing. Dynamic New Directions for the 1990's, Homewood, Business One Irwin.

BolwIJN, P.T. et T. KUMPE (1990), «Manufacturing in the 1990's - productivity, flexibility and innovation », Long Range Planning, vol. 23, $\mathrm{n}^{\circ} 4$, p. 44-57.

BUFFA, E.S. (1984), Meeting the Competitive Challenge, Homewood, Dow Jones-Irwin.

CHEN, W.H. (1999), «Manufacturing strategies of the network-based small firms : observations on the textile industry in Taiwan », Journal of Small Business Management, vol. $36, n^{\circ} 2$, p. 46-62. 
CHRISTOPHER, M. et RYALS (1999), «Supply chain strategy : it's impact on shareholder value », The International Journal of Logistics Management, vol. 10, $\mathrm{n}^{\circ} 1, \mathrm{p} .1-10$.

CoRbeTt, C. et L.V. WASSENHOVE (1993), «Trade-offs ? What trade-offs ? Competence and competitiveness in manufacturing strategy », California Management Review, été, p. 107-122.

DE MEYER, A. (1992), «An empirical investigation of manufacturing strategies in European industry », dans C.A. Voss (dir.), Manufacturing Strategy. Process and Content, Londres, Chapman \& Hall.

DE MEYer, A. et A. WitTEnBERG-Cox (1994), Nuevo enfoque de la función de producción, Barcelone, Folio.

De Meyer, A. J. NAKane, J.G. Miller, et K. Ferdows (1989), «Flexibility : the next competitive battle. The manufacturing futures survey», Strategic Management Journal, vol. 10, p. 135-144.

DYER, J.H. et H. SINGH (1998), «The relational view : cooperative strategy and sources of interorganizational competitive advantage », Academy and Management Review, vol. $23, \mathrm{n}^{\circ} 4$, p. $660-679$.

FINE, C. et A. HAX (1985), «Manufacturing strategy : a methodology and illustration », Interfaces, vol. 15, $\mathrm{n}^{\circ}$ 6, p. 28-46.

GARVIN, D.A. (1993), «Manufacturing strategy planning», California Management Review, été, p. 85-106.

GreENHALGH, G.R. (1990), Manufacturing Strategy. Formulation and Implementation, Sydney, Addison-Wesley.

HAAS, E.A. (1987), «Breakthrough manufacturing », Harvard Business Review, mars-avril, p. 75-81.

HARRISON, M. (1993), Operations Management Strategy, Londres, Pitman Publishing.

HAYeS, R.H. et S.C. WheElWright (1984), Restoring Our Competitive Edge, New York, John Wiley.

Hayes, R.H., S.C. Wheelwright et K.B. Clark (1988), Dynamic Manufacturing. Creating the Learning Organization, New York, The Free Press.

HILL, T.J. (1993), Manufacturing Strategy. The Management of the Manufacturing Function, Londres, MacMillan.

LAMBerT, D.M., M.C. CoOPER et J.D. PAGH (1998), «Supply chain management : implementation issues and research opportunities », The International Journal of Logistics Management, vol. 9, n 2, p. 1-19.

LAWRENCE, P. et J. LORSCH (1967), Organization and Environment, Boston, Harvard Business School Press.

LEONG, G.K., D.L. SNYDER et P.T. WARD (1990), « Research in the process and content of manufacturing strategy », OMEGA, vol. $18, \mathrm{n}^{\circ} 2$, p. 109-122.

Lehmann, D. (1979), Market Research and Analysis, Illinois, Homewood.

MARShall, A. (1969), Principles of Economics, Londres, MacMillan. 
MATTHEWS, C.H. et S.G. SCOTT (1995), « Uncertainty and planning in small and entrepreneurial firms : an empirical assessment », Journal of Small Business Management, vol. 33, $n^{\circ} 4$, p. 34-52.

MILler, S.S. (1988), Competitive Manufacturing : Using Production as a Management Tool, Wokingham, Van Nostrand Reinhold.

MiLleR, J.G. et A.V. ROTH (1988), «Manufacturing strategies : executive summary of the 1987 North American Manufacturing Future Survey », Operations Management Review, vol. 6, $\mathrm{n}^{\circ}$ 1, p. 8-20.

MilleR, J.G. et A.V. ROTH (1994), « A taxonomy of manufacturing strategies », Management Science, vol. 40, no 3, p. 285-304.

Mills, J., K. PlatTS et M. GREGORY (1995), «A framework for the design of manufacturing strategy processes. A contingency approach », International Journal of Operations \& Production Management, vol. 15, $\mathrm{n}^{\circ} 4$, p. 17-49.

MORRISON, A.J. et K. ROTH (1992), «A taxonomy of business-level strategies in global industries », Strategic Management Journal, vol. 13, p. 399-418.

NORUSIS, M.J. (1994), SPSS Professional Statistics 6.1, Illinois, SPSS Inc.

PlatTS, K.W. et M.J. GREGORY (1990), « Manufacturing audit in the process of strategy formulation », International Journal of Operations and Production Management, vol. $10, \mathrm{n}^{\circ}$ 9, p. 5-26.

PlatTS, K.W. et M.J. GreGORY (1992), «A manufacturing audit approach to strategy formulation », dans C.A. Voss (dir.), Manufacturing Strategy. Process and Content, Londres, Chapman \& Hall.

POWELL, T.C. (1992), «Organizational alignment as competitive advantage », Strategic Management Journal, vol. 13, n 2 , p. 119-134.

POWELL, T.C. (1996), « How much does industry matter ? An alternative empirical test », Strategic Management Journal, vol. 17, p. 323-334.

REGER, R.K. et A.S. HUFF (1993), «Strategic groups : a cognitive perspective », Strategic Management Journal, vol. 14, nº 2, p. 103-124.

RICHARDSON, P.R., A.J. TAYLOR et J.J. GORDON (1985), « A strategic approach to evaluating manufacturing performance », Interfaces, vol. 15, $\mathrm{n}^{\circ} 16$, p. 15-27.

ROBINSON, R. et J. PEARCE (1984), «Research thrusts in small firm strategic planning », Academy of Management Review, vol. 9, p. 128-137.

SCHMENNER, R.W. (1990), «The seven deadly sins of manufacturing », dans P.E. Moody (dir.), Strategic Manufacturing. Dynamic New Directions for the 1990's, Homewood, Ill., Business One Irwin.

SCHROEDER, R.G. et T.N. LAHR (1990), « Development of manufacturing strategy : a proven process », dans J.E. Ettlie, M.C. Burnstein et A. Fiegenbaum (dir.), Manufacturing Strategy: The Research Agenda for the Next Decade, Norwell, Kluwer Academic.

SKINNER, W. (1969), «Manufacturing : missing link in corporate strategy », Harvard Business Review, mai-juin, p. 136-145. 
SKINNER, W. (1985), Manufacturing : The Formidable Competitive Weapon, New York, John Wiley \& Sons.

STOBAUCH, R. et P. TELESIO (1983), « Match manufacturing policies and product strategy », Harvard Business Review, vol. 61, n 2, p. 113-120.

SWEENEY, M. et M. SZWEJCZEWSKI (1996), "The search for genetic manufacturing strategies in the UK engineering industry », Document de travail SWP 4/96, Cranfield, Cranfield School of Management.

SwINK, M. et M. HegarTy (1998), «Core manufacturing capabilities and their links to product differentiation ", International Journal of Operations and Production Management, vol. 18, $\mathrm{n}^{\circ}$ 4, p. 374-396.

TOSI, H., R. AldaG et R. STOREY (1973), «On the measurement of the environment : an assessment of Lawrence and Lorsch's environmental uncertainty scale », Administrative Science Quarterly, vol. 18, p. 27-36.

Voss, C.A. (1989), «Strategic approaches to manufacturing », The International Management Development Review, Bruxelles, Management Centre Europe.

Voss, C.A. (1992), « Manufacturing strategy formulation as a process », dans C.A. Voss (dir.), Manufacturing Strategy. Process and Content, Londres, Chapman \& Hall.

WHEELWRIGHT, S.C. (1978), « Reflecting corporate strategy in manufacturing decisions », Business Horizons, février.

WU, S.H. (1994), «Industrial manager and cooperative network systems », Taiwan Economic Research Monthly, vol. 17, $\mathrm{n}^{\circ}$ 10, p. 21-23.

YBARRA, J.A. (1992), «Entre la cooperación y la competencia : los distritos industriales en el país valenciano ", Economía Industrial, juillet-août, p. 72-74. 


\section{Annexe I}

Politiques de production. Les gestionnaires interrogés ont confirmé s'ils ont eu recours ou non, ces quatre dernières années, aux pratiques, aux politiques ou aux technologies suivantes : lignes de transfert, robots, manipulateurs, $\mathrm{CN}$, CAO, FAO, CAO / FAO, codes-barres, PBM, EDI avec les fournisseurs, EDI avec les clients, division de l'usine en usines plus petites, intégration verticale en amont, sous-traitance pour toute la production, sous-traitance d'une partie importante de la production, sous-traitance d'une faible partie de la production, changement de l'emplacement vers des zones moins industrialisées, changement de l'emplacement vers des zones plus industrialisées, utilisation d'une technologie de pointe, développement d'une technologie et de procédés propres, augmentation de la capacité de production, réduction de la capacité de production, JAT, GIQ, obtention de la certification AENOR de qualité du produit, certification ISO-9000, cercles de qualité, contrôle statistique des procédés, inspection systématique du produit fini, réalisation d'inspections pendant les phases intermédiaires du processus de production, réalisation d'inspections visuelles, réalisation d'inspections automatiques, réalisation d'inspections effectuées par des spécialistes du contrôle de la qualité ou faites en laboratoire, utilisation de systèmes très informatisés pour la gestion de la production, utilisation de systèmes peu informatisés pour la gestion de la production, utilisation de systèmes de point de commande pour la gestion des matières premières, utilisation de systèmes de point de commande pour la gestion de produits en cours de fabrication, utilisation de systèmes de point de commande pour la gestion des produits finis, cartes kanban, participation du personnel de R-D au développement du produit, participation du personnel de production au développement du produit, participation du personnel de marketing au développement du produit, participation du personnel de contrôle de la qualité au développement du produit, participation des fournisseurs au développement du produit, participation des clients au développement du produit.

Contexte de l'entreprise. Les gestionnaires interrogés ont évalué l'évolution d'une série de mesures du rendement au cours des quatre dernières années sur une échelle de cinq points, le 1 dans chaque variable indiquant que la situation s'est beaucoup dégradée et le 5, que la situation s'est beaucoup améliorée. Les mesures des entreprises ont visé les aspects suivants : la rentabilité de l'entreprise, la compétitivité de l'entreprise et le fonctionnement général de l'usine. Les mesures suivantes ont été évaluées sur une échelle de 
cinq points où (1) est inférieur ou égal à 2 jours, (2) signifie de 3 à 7 jours, (3) signifie de 8 à 14 jours, (4) signifie de 15 à 30 jours et (5) signifie plus de 30 jours : temps moyen entre le moment où une commande est faite au fournisseur jusqu'à la réception du matériel, temps moyen entre deux envois consécutifs du même fournisseur, quantité minimale ou réserve de matière première (en temps), quantité minimale ou réserve de produits finis (en temps), temps moyen qui s'écoule entre deux envois consécutifs au même client, temps moyen qui s'écoule entre l'instant où le client passe une commande et la réception de la marchandise.

Pour caractériser le type de procédé de production employé, nous avons proposé six autres variables dans lesquelles le gestionnaire devait se situer sur une échelle de sept points où le 1 et le 7 représentaient, pour chaque variable, les valeurs extrêmes suivantes : (1) nous fabriquons un petit volume d'une grande variété de produits et (7) nous fabriquons un grand volume de produits très standardisés; (1) les équipes sont formées autour de fonctions ou d'ateliers et (7) les équipes se relaient de façon séquentielle; (1) les matériaux sont transformés en très petites séries à l'aide de machines et (7) les matériaux sont transformés en grandes séries ou lots ; (1) le flux de matériaux est complexe et très variable et (7) le flux de matériaux est fixe ; (1) les équipes sont très flexibles et (7) les équipes sont très spécialisées ; (1) nous fabriquons sur commande et (7) nous fabriquons pour entreposer.

Nous avons mesuré l'orientation exportatrice avec une échelle de cinq points où nous recueillons le pourcentage de ventes de produits à exporter : (1) $0 \%$; (2) de $1 \%$ à $20 \%$; (3) de $21 \%$ à $40 \%$; (4) de $41 \%$ à $60 \%$; (5) de $61 \%$ à $100 \%$.

Le pourcentage d'utilisation de la capacité de production apparaît avec une variable mesurée sur une échelle de cinq points : (1) moins de $70 \%$; (2) de $70 \%$ à $79 \%$; (3) de $80 \%$ à $89 \%$; (4) de $90 \%$ à $94 \%$; (5) de $95 \%$ à $100 \%$.

Le nombre d'employés a été mesuré sur une échelle de cinq points : (1) de 0 à 50 travailleurs; (2) de 51 à $100 ;$ (3) de 101 à $150 ;$ (4) de 151 à 200 ; (5) plus de 200.

Le volume de ventes apparaît avec une variable mesurée sur une échelle de cinq points : (1) jusqu'à 1000 millions de pesetas; (2) de 1001 à 2000 ; (3) de 2001 à 3000 ; (4) de 3001 à 4000 ; (5) plus de 4000 . 